\title{
VALIDAÇÃO DO MODELO ClimaBR EM RELAÇÃO AO NÚMERO DE DIAS CHUVOSOS E À PRECIPITAÇÃO TOTAL DIÁRIA
}

\author{
SIDNEY S. ZANETTI ${ }^{1}$, VICENTE DE P. S. DE OLIVEIRA ${ }^{2}$, FERNANDO F. PRUSKI ${ }^{3}$
}

RESUMO: Este trabalho foi realizado com o objetivo de validar o modelo ClimaBR no que se refere à geração de séries sintéticas de precipitação total diária em diferentes localidades brasileiras. Para tanto, foram geradas séries sintéticas de precipitação para 12 localidades, escolhidas de forma distribuída, para que todas as diferentes regiões de precipitação homogênea do País fossem contempladas. A partir das séries pluviométricas, disponíveis nas estações meteorológicas das localidades selecionadas, o ClimaBR foi utilizado para gerar as séries sintéticas de precipitação, sendo gerada uma série de 100 anos para cada localidade. A qualidade dos resultados foi analisada comparando-se o ajuste entre os valores observados e os gerados pelo ClimaBR, por meio de regressão linear simples e de outros indicadores estatísticos. Os resultados obtidos permitem afirmar que, de acordo com as condições consideradas no presente estudo, o modelo ClimaBR apresentou bom desempenho na geração de séries sintéticas de precipitação (número de dias chuvosos e precipitação total diária) para todas as localidades testadas.

PALAVRAS-CHAVE: chuva, simulação de dados climáticos, séries sintéticas.

\section{VALIDATION OF THE MODEL ClimaBR IN RELATION TO THE NUMBER OF WET DAYS AND TO DAILY TOTAL PRECIPITATION}

ABSTRACT: This study was accomplished with the objective of validate the ClimaBR model in what refers to generate synthetic series of total daily rainfall in different Brazilian places. For that, synthetic series of rainfall were generated for 12 places, chosen in a distributed way, so that all the different homogeneous rainfall Brazilian's regions were observed. Starting from the pluviometric series, available in the meteorological stations from selected places, the ClimaBR was used to generate the synthetic series of rainfall; it was generated 100-year-old series to each place. The results quality was analyzed comparing the adjustment among the observed values and the generated values by ClimaBR, through simple lineal regression and of other statistical indicators. The results obtained allow affirming that, in agreement with the conditions considered in the present study, the software ClimaBR presented good acting to generate of synthetic series of precipitation (number of wet days and daily total rainfall) for all of the tested places.

KEYWORDS: rainfall, simulation of climatic data, synthetic series.

\section{INTRODUÇÃO}

A precipitação pluvial é um dos elementos climáticos que exerce maior influência sobre as condições ambientais. Além do seu efeito direto no balanço hídrico, também interfere indiretamente em outros elementos climáticos, como nas temperaturas do ar e do solo, na umidade do ar e na radiação solar incidente que, em conjunto, atuam como fatores básicos no comportamento dos sistemas hidrológicos.

\footnotetext{
${ }^{1}$ Eng o Agrônomo, Doutorando em Produção Vegetal, Laboratório de Engenharia Agrícola, Universidade Estadual do Norte Fluminense, Campos dos Goytacazes - RJ, Fone: (0XX22) 2726.1543, sidney@uenf.br, Bolsista do CNPq.

${ }^{2}$ Eng ${ }^{\mathrm{O}}$ Agrimensor, Prof. Doutor, Centro Federal de Educação Tecnológica de Campos, Campos dos Goytacazes - RJ.

${ }^{3}$ Eng ${ }^{o}$ Agrícola, Prof. Titular, Departamento de Engenharia Agrícola, Universidade Federal de Viçosa, Viçosa - MG, Bolsista do CNPq. Recebido pelo Conselho Editorial em: 27-10-2004

Aprovado pelo Conselho Editorial em: 9-2-2006
} 
Dados pluviométricos disponíveis e confiáveis são fundamentais para uma eficaz previsão de eventos impactantes ao meio ambiente. Um fator limitante para a utilização de modelos hidrológicos é a disponibilidade de dados climáticos. Quando esses dados são inexistentes e/ou inadequados, é comum a geração de uma série sintética produzida por modelo estocástico. Uma série sintética é o resultado da associação de séries de dados reais com números aleatórios produzidos por algoritmos computacionais, a fim de gerar seqüências de números aleatórios que se assemelham aos dados climáticos reais (WILKS, 1999). Para o Brasil, a aplicação desses procedimentos apresenta grande potencial, tendo em vista o fato de a extensão das séries históricas disponíveis normalmente ser reduzida, principalmente quando se trata de dados pluviográficos.

Dentre os modelos estrangeiros mais utilizados para a geração de séries sintéticas de elementos climáticos, podem ser citados o WGEN - Weather Generation (RICHARDSON, 1981), o CLIGEN Climate Generator (NICKS et al., 1995), o GEM - Generation of Weather Elements for Multiple Applications (JOHNSON, 2003) e, no Brasil, o ClimaBR - Gerador de Séries Sintéticas de Precipitação (OLIVEIRA, 2003; ZANETTI et al., 2005) e o SEDAC_R - Simulador Estocástico de Dados Climáticos (VIRGENS FILHO, 2001).

O modelo ClimaBR (http://www.ufv.br/dea/gprh/climabr) foi desenvolvido a partir da aplicação de princípios estatísticos e matemáticos associados às informações pluviográficas e pluviométricas de 11 estações meteorológicas situadas no Estado do Rio de Janeiro. As probabilidades condicionais para a ocorrência de dias chuvosos são estimadas mensalmente pelo processo estocástico denominado cadeia de Markov, que utiliza dependências de primeira ordem com apenas dois estados. Para os dias considerados chuvosos, a lâmina total precipitada é estimada a partir da distribuição Pearson tipo III, com diferentes parâmetros para cada mês do ano. Com o ClimaBR, é possível a geração de séries sintéticas de precipitação, incluindo, além da precipitação total diária, a sua duração, o tempo padronizado de ocorrência da intensidade máxima instantânea e a própria intensidade máxima instantânea dos eventos. Com essas informações, é possível obter, também, o perfil instantâneo de precipitação (OLIVEIRA, 2003; ZANETTI et al., 2005). Entretanto, esses autores afirmam que a estimação de algumas dessas variáveis, necessárias para a obtenção do perfil de precipitação, precisa ser melhorada no modelo.

O ClimaBR continua em fase de desenvolvimento pelo Grupo de Pesquisa em Recursos Hídricos do Departamento de Engenharia Agrícola da Universidade Federal de Viçosa (GPRH/DEA/UFV), onde está sendo feito o aprimoramento das simulações referentes à duração e à intensidade máxima de precipitação, bem como está sendo adicionada a possibilidade de simulação de outros elementos climáticos, como a radiação solar, a velocidade do vento, a umidade relativa e as temperaturas máxima e mínima do ar.

Devido à importância das séries temporais de precipitação, vários pesquisadores vêm se empenhando em obter técnicas que permitam a geração de dados sintéticos que realmente representem o padrão de chuvas de determinada região. Sendo assim, torna-se necessário que esses dados simulados passem por uma avaliação, a fim de analisar a sua confiabilidade e a representação das condições climáticas reais do local de interesse.

Diante do exposto e ressaltando que o ClimaBR foi desenvolvido e testado levando-se em consideração somente 11 localidades do Estado do Rio de Janeiro, teve-se o objetivo de, no presente trabalho, validar o modelo ClimaBR no que se refere à simulação do número de dias chuvosos e da lâmina de precipitação total diária para diferentes localidades brasileiras. 


\section{MATERIAL E MÉTODOS}

O modelo ClimaBR, por meio de seu respectivo programa computacional, foi utilizado para gerar séries sintéticas de precipitação diária para 12 localidades brasileiras, a partir de séries históricas pluviométricas das mesmas. As estações meteorológicas (localidades) foram escolhidas de forma distribuída, considerando-se dois critérios. O primeiro refere-se à localização espacial das estações, a qual foi orientada a partir da classificação do território brasileiro em regiões homogêneas de precipitação, conforme apresentado por BAENA (2004). Tal classificação foi adaptada do estudo realizado por SILVA (2004), que considerou apenas a distribuição do total precipitado para identificar as áreas homogêneas. Como segundo critério, foi considerado o tamanho das séries históricas, ou seja, dentro de cada região foram selecionadas as estações com maior número de anos de registros pluviométricos.

Para realizar as simulações, foram utilizadas 12 séries históricas de precipitação diária, cada uma proveniente de uma localidade, conforme apresentadas na Tabela 1 e na Figura 1.

Uma vez obtidos os dados das estações, o ClimaBR foi utilizado para gerar as séries sintéticas de precipitação, sendo gerada uma série de 100 anos para cada localidade. Em cada série sintética gerada, apenas os dados referentes à precipitação total diária foram analisados.

Visando a possibilitar a comparação entre os valores observados e gerados pelo ClimaBR, foram calculados, tanto para as séries históricas quanto para as séries sintéticas, as seguintes informações: a média $\left(\mathrm{x}_{\mathrm{j}}, \mathrm{mm}\right)$, o desvio-padrão $\left(\mathrm{s}_{\mathrm{j}}, \mathrm{mm}\right)$ e o coeficiente de assimetria $\left(\mathrm{g}_{\mathrm{j}}\right.$, adimensional) mensal da precipitação total diária; a probabilidade, obtida para cada mês, de ocorrência de um dia chuvoso sendo o dia anterior chuvoso $\left[\mathrm{P}(\mathrm{C} / \mathrm{C})_{\mathrm{j}}\right.$, decimal]; a probabilidade, obtida para cada mês, de ocorrência de um dia chuvoso sendo o dia anterior seco $\left[\mathrm{P}(\mathrm{C} / \mathrm{S})_{\mathrm{j}}\right.$, decimal $]$, e o número médio de dias chuvosos em cada mês $\left(\mathrm{DC}_{\mathrm{j}}\right.$, dias $)$.

Para cada uma dessas informações, foi realizada uma regressão linear simples ( $\left.Y=\beta_{0}+\beta_{1} X\right)$, sendo considerada como variável independente os valores mensais observados e como variável dependente os valores mensais obtidos a partir da série sintética gerada pelo ClimaBR. Os resultados foram analisados por meio dos coeficientes $\beta_{\mathrm{o}}, \beta_{1}$ e $\mathrm{r}^{2}$ das equações. O teste " $\mathrm{t}$ " foi utilizado para avaliar estatisticamente o valor do intercepto $\left(\right.$ Ho: $\left.\beta_{\mathrm{o}}=0\right)$ e da inclinação da reta $\left(\right.$ Ho: $\left.\beta_{1}=1\right)$, a $1 \%$ de probabilidade (significância).

TABELA 1. Estações meteorológicas utilizadas na validação do ClimaBR (dados pluviométricos).

\begin{tabular}{lccccc}
\hline Nome da Estação & Estado & Código & $\begin{array}{c}\text { Latitude } \\
\text { (sul) }\end{array}$ & $\begin{array}{c}\text { Longitude } \\
\text { (oeste) }\end{array}$ & $\begin{array}{c}\text { Número de Anos } \\
\text { de Registros }\end{array}$ \\
\hline Abelardo Luz & SC & 02652000 & $26^{\circ} 33^{\prime} 22^{\prime \prime}$ & $52^{\circ} 19^{\prime} 51^{\prime \prime}$ & 43 \\
Açude Bonito & RN & 00638044 & $06^{\circ} 12^{\prime} 46^{\prime \prime}$ & $38^{\circ} 25^{\prime} 32^{\prime \prime}$ & 35 \\
Campo dos Cavalos & BA & 00940018 & $09^{\circ} 33^{\prime} 35^{\prime \prime}$ & $40^{\circ} 38^{\prime} 48^{\prime \prime}$ & 48 \\
Estrada do Quata & SP & 02250001 & $22^{\circ} 03^{\prime} 48^{\prime \prime}$ & $50^{\circ} 36^{\prime} 34^{\prime \prime}$ & 29 \\
Estrela do Norte & GO & 01349000 & $13^{\circ} 52^{\prime} 18^{\prime \prime}$ & $49^{\circ} 04^{\prime} 17^{\prime}$, & 24 \\
Jaciguá (DNOS) & ES & 02041010 & $20^{\circ} 42^{\prime} 07^{\prime \prime}$ & $41^{\circ} 00^{\prime} 59^{\prime \prime}$ & 42 \\
Leal de Patos & MG & 01846017 & $18^{\circ} 38^{\prime} 28^{\prime \prime}$ & $46^{\circ} 20^{\prime} 04^{\prime \prime}$ & 26 \\
Rondonópolis & MT & 01654000 & $16^{\circ} 28^{\prime} 14^{\prime \prime}$ & $54^{\circ} 39^{\prime} 23^{\prime \prime}$ & 31 \\
Santa Isabel do Pará & PA & 00148003 & $01^{\circ} 17^{\prime} 49^{\prime \prime}$ & $48^{\circ} 10^{\prime} 15^{\prime \prime}$ & 19 \\
Santa Maria do Boiacu & RR & 00061000 & $00^{\circ} 30^{\prime} 19^{\prime \prime}$ & $61^{\circ} 47^{\prime} 11^{\prime \prime}$ & 25 \\
Santarém & PA & 00254000 & $02^{\circ} 25^{\prime} 56^{\prime \prime}$ & $54^{\circ} 41^{\prime} 27^{\prime \prime}$ & 25 \\
São Lourenço do Sul & RS & 03151003 & $31^{\circ} 22^{\prime} 10^{\prime \prime}$ & $51^{\circ} 59^{\prime} 12^{\prime \prime}$ & 43 \\
\hline
\end{tabular}




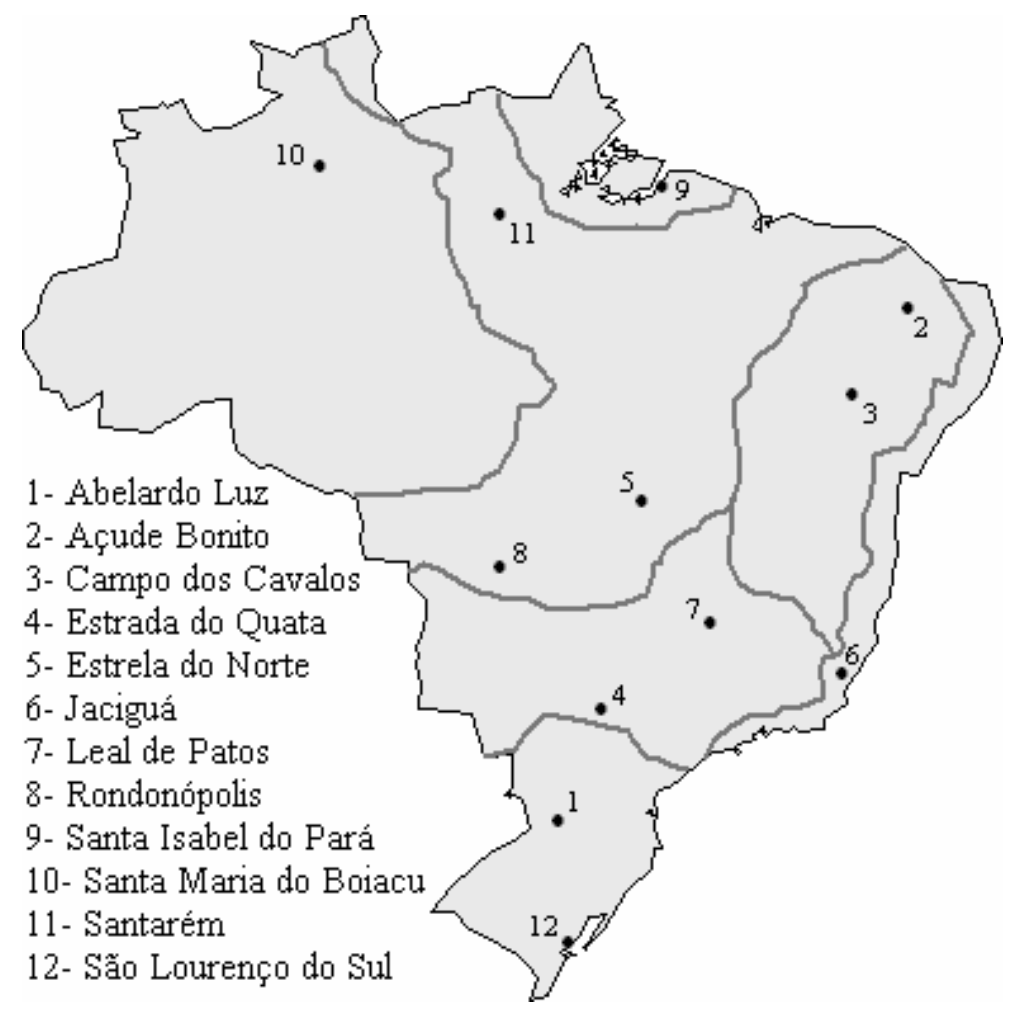

FIGURA 1. Distribuição espacial das estações meteorológicas utilizadas na validação do modelo ClimaBR (baseada nas regiões homogêneas de precipitação).

Adicionalmente, para aferir o grau de concordância entre valores observados e estimados, foi calculado o erro-padrão de estimativa (EPE) e o índice de concordância (d) apresentado por WILLMOTT (1981), sendo obtidos pela aplicação das eqs. (1) e (2), respectivamente.

$$
\begin{aligned}
& \operatorname{EPE}=\left(\frac{\sum_{\mathrm{i}=1}^{\mathrm{n}}\left(\hat{\mathrm{y}}_{\mathrm{i}}-\mathrm{y}_{\mathrm{i}}\right)^{2}}{\mathrm{n}-1}\right)^{0,5} \\
& \mathrm{~d}=1-\frac{\sum_{\mathrm{i}=1}^{\mathrm{n}}\left(\hat{\mathrm{y}}_{\mathrm{i}}-\mathrm{y}_{\mathrm{i}}\right)^{2}}{\sum_{\mathrm{i}=1}^{\mathrm{n}}\left(\left|\hat{\mathrm{y}}_{\mathrm{i}}-\overline{\mathrm{y}}\right|+\left|\mathrm{y}_{\mathrm{i}}-\overline{\mathrm{y}}\right|\right)^{2}}
\end{aligned}
$$

em que,

$\mathrm{y}_{\mathrm{i}}$ - valor mensal observado;

$\hat{y}_{\mathrm{i}}$ - valor mensal estimado;

$\mathrm{n}$ - número de observações, 12 valores, e

$\bar{y}$ - média aritmética dos valores mensais observados. 
$\mathrm{O}$ índice d é um valor adimensional, variando entre 0 e 1 , sendo que o valor 1 representa o completo ajustamento, enquanto o valor 0 indica o oposto. Nas eqs. (1) e (2), todas as variáveis, exceto d e n, que são adimensionais, assumem a unidade da informação que será analisada em cada momento.

Visando a simplificar a apresentação dos resultados, foi selecionada apenas uma das localidades para apresentação dos mesmos, pois, por meio de observação prévia dos resultados, verificou-se que o desempenho do ClimaBR foi similar em todos os locais. Portanto, foi selecionada Rondonópolis - MT, cujos resultados obtidos para essa localidade são considerados representativos das demais. Somente os resultados referentes ao índice de concordância d são apresentados para todas as localidades e informações estudadas.

\section{RESULTADOS E DISCUSSÃO}

Na Tabela 2, estão apresentados os indicadores estatísticos do ajuste entre os valores observados e gerados pelo ClimaBR para a localidade de Rondonópolis. Os valores descritos referem-se à média, desvio-padrão e coeficiente de assimetria da precipitação total diária, às probabilidades de ocorrência de chuva e ao número médio mensal de dias chuvosos.

TABELA 2. Indicadores estatísticos referentes ao ajustamento entre dados observados (31 anos) e gerados pelo ClimaBR (100 anos) para a localidade de Rondonópolis - MT.

\begin{tabular}{lccccc}
\hline \multicolumn{1}{c}{ Descrição } & $\beta_{\mathrm{o}}$ & $\beta_{1}$ & $\mathrm{r}^{2}$ & EPE* & $\mathrm{d}$ \\
\hline Média $\left(\mathrm{x}_{\mathrm{j}}\right)$ & 0,164 & 0,992 & 0,9977 & 0,14 & 0,9992 \\
Desvio-Padrão $\left(\mathrm{s}_{\mathrm{j}}\right)$ & $-0,248$ & 1,012 & 0,9991 & 0,12 & 0,9996 \\
Coeficiente de assimetria $\left(\mathrm{g}_{\mathrm{j}}\right)$ & $-0,153$ & 1,122 & 0,8600 & 0,46 & 0,9439 \\
$\mathrm{P}(\mathrm{C} / \mathrm{C})_{\mathrm{j}}$ & $-0,002$ & 0,997 & 0,9691 & 0,03 & 0,9920 \\
$\mathrm{P}(\mathrm{C} / \mathrm{S})_{\mathrm{j}}$ & 0,007 & 0,960 & 0,9955 & 0,01 & 0,9984 \\
Dias chuvosos $\left(\mathrm{DC}_{\mathrm{j}}\right)$ & $-0,287$ & 1,014 & 0,9913 & 0,60 & 0,9975 \\
\hline
\end{tabular}

* EPE segue a unidade da variável que está sendo analisada em cada linha da tabela.

Para a localidade apresentada na Tabela 2, assim como para todas as demais localidades, os coeficientes $\beta_{\mathrm{o}}$ e $\beta_{1}$ não diferiram estatisticamente de 0 e 1 , respectivamente, pelo teste "t", a $1 \%$ de probabilidade. Isso permite inferir que os valores gerados pelo ClimaBR não diferem dos valores observados. O bom ajuste pode ser confirmado pelos baixos valores do EPE e pelos altos valores de $\mathrm{d}$ e $\mathrm{r}^{2}$ apresentados.

Na Figura 2, são apresentados os valores do índice de concordância (d) entre os dados observados e os gerados pelo ClimaBR para as 12 localidades que foram testadas. Pode ser observado que $89 \%$ dos índices calculados situam-se acima de 0,9 . Os $11 \%$ restantes referem-se aos valores obtidos a partir do coeficiente de assimetria, os quais variaram entre 0,7362 e 0,9439, apresentando somente quatro valores acima de 0,9 . Os índices de concordância calculados para o coeficiente de assimetria, apesar de não terem proporcionado a mesma ordem de magnitude que os demais, são considerados satisfatórios, visto que as regiões consideradas no presente estudo demonstraram grande variabilidade entre si, no que se refere às características pluviométricas regionais.

Pelos dados das séries históricas, foi constatado que, em uma das estações do Nordeste do País (Campo dos Cavalos), nos meses de julho, agosto e setembro, não houve nenhum dia chuvoso seguido de outro dia chuvoso em toda a série histórica (48 anos), ou seja, não existiram dois dias consecutivos com ocorrência de precipitação. Portanto, o valor observado de $\mathrm{P}(\mathrm{C} / \mathrm{S})_{\mathrm{j}}$ é muito baixo, devido ao reduzido número de dias chuvosos, e o valor de $\mathrm{P}(\mathrm{C} / \mathrm{C})_{\mathrm{j}}$ é nulo. Mesmo com esse tipo de condição 
adversa, o modelo ClimaBR apresentou bom desempenho, gerando série sintética de precipitação similar à série histórica observada.

TABELA 3. Índice de concordância (d) entre dados observados e gerados pelo ClimaBR para as 12 estações meteorológicas (localidades) testadas.

\begin{tabular}{lcccccc}
\hline \multirow{2}{*}{ Nome da Estação } & \multicolumn{7}{c}{ Índice de Concordância (d) } \\
\cline { 2 - 7 } & $\mathrm{x}_{\mathrm{j}}$ & $\mathrm{s}_{\mathrm{j}}$ & $\mathrm{g}_{\mathrm{j}}$ & $\mathrm{P}(\mathrm{C} / \mathrm{C})_{\mathrm{j}}$ & $\mathrm{P}(\mathrm{C} / \mathrm{S})_{\mathrm{j}}$ & $\mathrm{DC}_{\mathrm{j}}$ \\
\hline Abelardo Luz & 0,9999 & 0,9999 & 0,9228 & 0,9648 & 0,9811 & 0,9080 \\
Açude Bonito & 0,9994 & 0,9998 & 0,7891 & 0,9941 & 0,9973 & 0,9962 \\
Campo dos Cavalos & 0,9988 & 0,9996 & 0,7387 & 0,9871 & 0,9991 & 0,9946 \\
Estrada do Quatá & 0,9994 & 0,9996 & 0,8545 & 0,9818 & 0,9969 & 0,9964 \\
Estrela do Norte & 0,9999 & 1,0000 & 0,8303 & 0,9986 & 0,9990 & 0,9988 \\
Jaciguá (DNOS) & 0,9999 & 0,9999 & 0,8906 & 0,9814 & 0,9950 & 0,9871 \\
Leal de Patos & 1,0000 & 1,0000 & 0,7821 & 0,9943 & 0,9990 & 1,0000 \\
Rondonópolis & 0,9992 & 0,9996 & 0,9439 & 0,9920 & 0,9984 & 0,9975 \\
Santa Isabel do Pará & 0,9999 & 0,9999 & 0,9381 & 0,9971 & 0,9911 & 0,9983 \\
Santa Maria do Boiacu & 0,9999 & 0,9999 & 0,9370 & 0,9963 & 0,9930 & 0,9930 \\
Santarém & 0,9995 & 0,9999 & 0,7394 & 0,9987 & 0,9981 & 0,9995 \\
São Lourenço do Sul & 0,9999 & 1,0000 & 0,7362 & 0,9476 & 0,9590 & 0,9524 \\
\hline
\end{tabular}

Também nas condições climáticas do Sul do País (São Lourenço do Sul), onde o número médio mensal de dias chuvosos é praticamente constante em todos os meses do ano, o desempenho do modelo foi igualmente satisfatório.

Tal comportamento do modelo ClimaBR comprova que a adoção do processo estocástico denominado cadeia de Markov, que utiliza dependências de primeira ordem para calcular as probabilidades condicionais de ocorrência de chuva $\left[\mathrm{P}(\mathrm{C} / \mathrm{C})_{j}\right.$ e $\left.\mathrm{P}(\mathrm{C} / \mathrm{S})_{\mathrm{j}}\right]$, proporcionou que as simulações da distribuição do número de dias chuvosos fossem representativas do que ocorre na realidade, mesmo em situações de grande variabilidade no comportamento climático (diferentes regiões homogêneas de precipitação). Esse fato, provavelmente, colabora para que a precipitação total diária também seja simulada com maior eficiência.

Em relação à precipitação total diária, os resultados (média e desvio-padrão mensal) para todas as demais localidades testadas foram similares aos que podem ser observados para Rondonópolis. $\mathrm{O}$ bom desempenho do ClimaBR pode ser justificado pelo algoritmo utilizado para calcular a lâmina de precipitação dos dias chuvosos, pois esse se baseia na distribuição Pearson tipo III (NICKS et al., 1995), além de duas outras equações adicionais propostas por OLIVEIRA (2003) e ZANETTI et al. (2005), as quais se apresentaram bastante eficientes. Essas equações realizam correções, tendo como finalidade melhorar o ajuste entre as precipitações totais diárias simuladas e as observadas nos dados reais (série histórica).

\section{CONCLUSÕES}

Os resultados obtidos permitem afirmar que, de acordo com os dados e com as condições consideradas no presente estudo, o modelo ClimaBR apresentou bom desempenho na geração de séries sintéticas de precipitação (número de dias chuvosos e precipitação total diária) para todas as localidades testadas, mesmo para as condições contrastantes existentes nas diferentes regiões do Brasil. Com esse desempenho, fica comprovado que o ClimaBR é um modelo apto para gerar séries sintéticas de precipitação tanto para o Rio de Janeiro (Estado para o qual o modelo foi desenvolvido) quanto para qualquer outra localidade brasileira. 


\section{REFERÊNCIAS}

BAENA, L.G.N. Modelo para geração de séries sintéticas de dados climáticos. 2004. 172 f. Tese (Doutorado em Recursos Hídricos e Ambientais) - Universidade Federal de Viçosa, Viçosa, 2004.

JOHNSON, G.J. Weather generator technology. Portland: United States Department of Agriculture, 2003. Disponível em: http://www.wcc.nrcs.usda.gov/water/climate/gem/gem.html. Acesso em: 2 fev. 2003.

NICKS, A.D.; LANE, L.J.; GANDER, G.A. Weather Generator: USDA-Water erosion prediction project (WEPP). West Lafayette: USDA-ARS National Soil Erosion Research Laboratory, 1995. Cap. $2,22 \mathrm{p}$.

OLIVEIRA, V.P.S. Modelo para a geração de séries sintéticas de precipitação. 2003. 156 f. Tese (Doutorado em Recursos Hídricos e Ambientais) - Universidade Federal de Viçosa, Viçosa, 2003.

RICHARDSON, C.W. Stochastic simulation of daily precipitation, temperature, and solar radiation. Water Resources Research, Washington, v.17, n.1, p.182-90, 1981.

SILVA, A.M. Rainfall erosivity map for Brazil. Catena, Oxford, v.57, n.3, p.251-9, 2004.

VIRGENS FILHO, J.S. Ferramenta computacional para simulação de séries climáticas diárias, baseada na parametrização dinâmica das distribuições de probabilidade. 2001. $92 \mathrm{f}$ Tese (Doutorado em Energia na Agricultura) - Faculdade de Ciências Agronômicas, Universidade Estadual Paulista, Botucatu, 2001.

WILKS, D.S. Simultaneous stochastic simulation of daily precipitation, temperature and solar radiation at multiple sites in complex terrain. Agricultural and Forest Meteorology, Amsterdam, v.96, n.1-3, p.85-101, 1999.

WILLMOTT, C.J. On the validation of model. Physical Geography, Los Angeles, v.2, n.2, p.184-94, 1981.

ZANETTI, S.S.; PRUSKI, F.F.; MOREIRA, M.C.; SEDIYAMA, G.C.; SILVA, D.D. Programa computacional para geração de séries sintéticas de precipitação. Engenharia Agrícola, Jaboticabal, v.25, n.1, p.96-104, 2005. 